Conference abstract POT08

\title{
Interactions Of Different Sartans with the Bilayer Interface Studied by Saxs
}

\author{
M. RAPPOLT $^{1}{ }^{1}$, B. SARTORI ${ }^{1}$, A. HodzIC ${ }^{1,2}$, G. PABSt ${ }^{1}$, T. MAVROMOUSTAKOS ${ }^{3}$ \\ ${ }^{1}$ Institute of Biophysics and Nanosystems Research, Austrian Academy of Sciences, 8042 Graz, Austria \\ ${ }^{2}$ Research Center Pharmaceutical Engineering GmbH, 8010 Graz, Austria \\ ${ }^{3}$ Chemistry Department, National and Kapodistrian University of Athens, Panepistimioupolis Zographou \\ 15771, Greece \\ E-mail: michael.rappolt@elettra.trieste.it (M. Rappolt)
}

Sci Pharm. 2010; 78: 723

doi:10.3797/scipharm.cespt.8.POT08

This work presents a thorough investigation of the interaction of the novel synthetic pyrrolidinone analog MMK3 and other SARTANS (losartan, valsartan and candesartan) with the model membrane system of dipalmitoylphosphatidylcholine (DPPC). SARTANS are designed to exert antihypertensive activity by functioning as an antagonist of the angiotensin II receptor of subtype $1\left(A T_{1}\right)$. Small angle X-ray scattering (SAXS) experiments on the interaction of SARTANS with DPPC bilayers were carried out and results demonstrate that all studied SARTANS are well incorporated into the membrane leaflets and furthermore cause partial bilayer interdigitation. Further structural as well as dynamical effects will be discussed, and compared to their overall efficiency as antihypertensive drug. 